\title{
Broadband non-polarizing terahertz beam splitters with variable split ratio
}

Minggui Wei, , Quan Xu, , Qiu Wang, , Xueqian Zhang, , Yanfeng Li, , Jianqiang Gu, , Zhen Tian, , Xixiang Zhang, , Jiaguang Han, and, and Weili Zhang

Citation: Appl. Phys. Lett. 111, 071101 (2017); doi: 10.1063/1.4986538

View online: http://dx.doi.org/10.1063/1.4986538

View Table of Contents: http://aip.scitation.org/toc/apl/111/7

Published by the American Institute of Physics

\section{Articles you may be interested in}

Magnetically-tunable cutoff in asymmetric thin metal film plasmonic waveguide

Applied Physics Letters 111, 071102 (2017); 10.1063/1.4991756

Active tuning of high- $Q$ dielectric metasurfaces

Applied Physics Letters 111, 053102 (2017); 10.1063/1.4997301

Optically active dilute-antimonide III-nitride nanostructures for optoelectronic devices

Applied Physics Letters 111, 061101 (2017); 10.1063/1.4997450

Near-infrared linewidth narrowing in plasmonic Fano-resonant metamaterials via tuning of multipole contributions

Applied Physics Letters 111, 061104 (2017); 10.1063/1.4997423

Cherenkov terahertz radiation from graphene surface plasmon polaritons excited by an electron beam Applied Physics Letters 110, 231102 (2017); 10.1063/1.4984961

A three-dimensional all-metal terahertz metamaterial perfect absorber

Applied Physics Letters 111, 051101 (2017); 10.1063/1.4996897

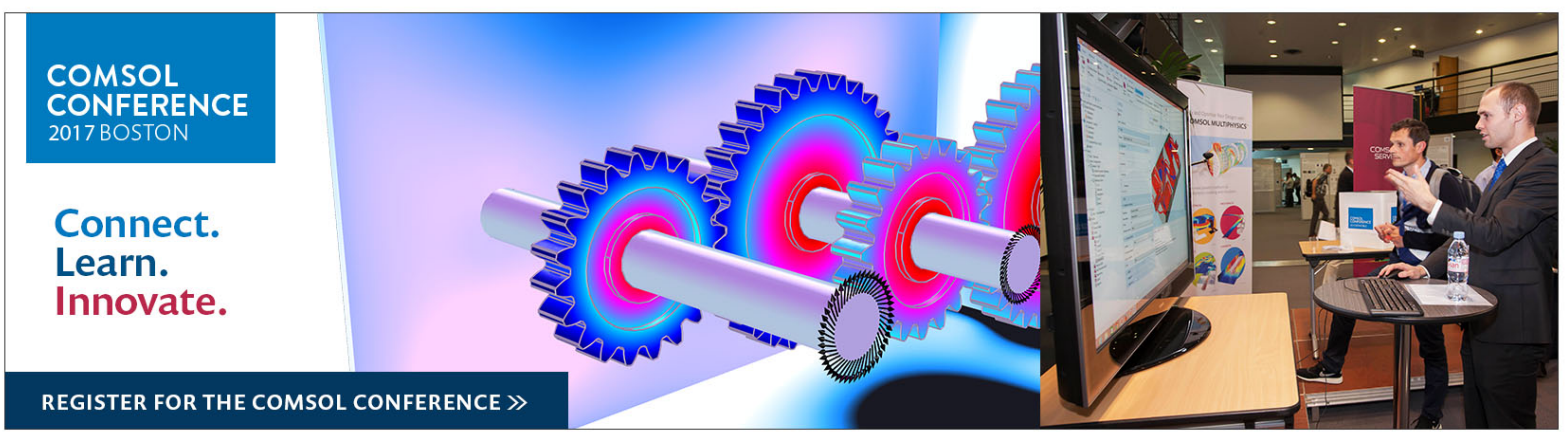




\title{
Broadband non-polarizing terahertz beam splitters with variable split ratio
}

\author{
Minggui Wei, ${ }^{1}$ Quan Xu, ${ }^{1}$ Qiu Wang, ${ }^{1}$ Xueqian Zhang, ${ }^{1}$ Yanfeng $\mathrm{Li},{ }^{1}$ Jianqiang Gu, ${ }^{1}$ \\ Zhen Tian, ${ }^{1, a)}$ Xixiang Zhang, ${ }^{2}$ Jiaguang Han, ${ }^{1, b)}$ and Weili Zhang ${ }^{1,3, c)}$ \\ ${ }^{1}$ Center for Terahertz waves and College of Precision Instrument and Optoelectronics Engineering, \\ Key Laboratory of Optoelectronics Information and Technical Science, Ministry of Education, \\ Tianjin University, Tianjin 300072, People's Republic of China \\ ${ }^{2}$ Division of Physical Science and Engineering, King Abdullah University of Science and Technology, \\ ThuWal 23955-6900, Saudi Arabia \\ ${ }^{3}$ School of Electrical and Computer Engineering, Oklahoma State University, Stillwater, Oklahoma 74078, USA
}

(Received 5 June 2017; accepted 30 July 2017; published online 15 August 2017)

\begin{abstract}
Seeking effective terahertz functional devices has always aroused extensive attention. Of particular interest is the terahertz beam splitter. Here, we have proposed, designed, manufactured, and tested a broadband non-polarizing terahertz beam splitter with a variable split ratio based on an alldielectric metasurface. The metasurface was created by patterning a dielectric surface of the $N$-step phase gradient and etching to a few hundred micrometers. The conversion efficiency as high as $81 \%$ under the normal incidence at $0.7 \mathrm{THz}$ was achieved. Meanwhile, such a splitter works well over a broad frequency range. The split ratio of the proposed design can be continuously tuned by simply shifting the metasurface, and the angle of emergences can also be easily adjusted by choosing the step of phase gradients. The proposed design is non-polarizing, and its performance is kept under different polarizations. Published by AIP Publishing. [http://dx.doi.org/10.1063/1.4986538]
\end{abstract}

Beam splitters are key components and widely used at various optical systems. In the optical realm, as a standard device, the beam splitter is well established, and it could be achieved by many methods, such as plasmonic waveguides, ${ }^{1}$ multimode interference structures, ${ }^{2}$ grating structures, ${ }^{3,4}$ directional couplers, ${ }^{5}$ multilayer films, ${ }^{6}$ and metamaterials. ${ }^{78}$ With the rapid development of terahertz technology, the terahertz frequency regime is still in great demand for highly efficient, flexible, and low-cost beam splitters. Specifically, in some complex terahertz systems, for instance, in the coherent terahertz measurements system, ${ }^{9}$ a split-ratio-variable splitter is desired to control the two-beam interference. However, due to the lack of natural materials with desired properties, e.g., the proper birefringence coefficient and low intrinsic material loss, it is challenging to build terahertz beam splitters with good performance. Among various polarizing terahertz beam splitter schemes, metallic wire-grating polarizing beam splitters are most commonly used. ${ }^{10-12}$ On the other hand, for the non-polarizing beam splitters, the pellicle and silicon are generally adopted in the terahertz system, ${ }^{13}$ which usually require oblique illumination, and the fabrication process of the pellicle splitter is also complicated because multiple layers of different materials are usually needed. In addition, the split ratio (SR) and the angle of emergence are often fixed for these nonpolarizing beam splitters. Generally, we define the SR here as

$$
S R=\frac{I_{\text {left }}}{I_{\text {right }}}
$$

where $I_{\text {left }}$ and $I_{\text {right }}$ represent the electric field intensities of the outgoing left and right beams, respectively.

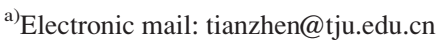

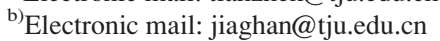

${ }^{\text {c) }}$ Electronic mail: weili.zhang@okstate.edu
}

A promising solution is the use of the metasurfaces, a planar metamaterial consisting of subwavelength plasmonic or dielectric structure, which is capable of tailoring the wavefront of outgoing electromagnetic waves in an extraordinary manner and is demonstrated well in the terahertz regime and other frequencies. ${ }^{14-16}$ Many exotic metasurface-based terahertz devices, such as terahertz lens, ${ }^{17,18}$ polarizers, ${ }^{19,20}$ and modulators, ${ }^{21,22}$ have been demonstrated previously. Inspired by this, in this letter, we established a broadband non-polarizing terahertz beam splitter with a variable SR based on an all-dielectric metasurface. Compared to metallic metasurfaces, the all-dielectric metasurfaces have significant improvement in conversion efficiency. ${ }^{23-25}$ Here, we use low-loss silicon structures and get the conversion efficiency as high as $81.22 \%$ at $0.7 \mathrm{THz}$, and the measured SR can be varied from 1.07 (48.31:51.69) to 301.6 (99.67:0.33). Meanwhile, such a splitter performs well over a broadband covering from $0.65 \mathrm{THz}$ to $0.95 \mathrm{THz}$. The proposed design is also independent of the polarization state of the incident beam, which can meet different application requirements. The metasurfacebased beam splitter with a variable SR can be assembled into the optical systems and thus assists in the realization of the convenient optical path design.

As illustrated in Fig. 1, the essence of the chosen splitter is an all-dielectric metasurface. The beam incidents normally on the metasurface and then is divided into two beams from the center point $O$. The angle of emergence of each beam $\theta_{1}$ or $\theta_{2}$ is determined by the formula ${ }^{14,26}$

$$
\begin{aligned}
& n_{t} \sin \theta_{1}=n_{i} \sin \theta_{i}-\frac{\lambda}{2 \pi} \frac{d \phi_{1}}{d x}, \\
& n_{t} \sin \theta_{2}=n_{i} \sin \theta_{i}+\frac{\lambda}{2 \pi} \frac{d \phi_{2}}{d x},
\end{aligned}
$$

where $\theta_{\mathrm{i}}$ is the incident angle; $\theta_{1}$ and $\theta_{2}$ are the angle of emergence of the left and right beams, respectively; $n_{\mathrm{i}}$ and $n_{\mathrm{t}}$ are 


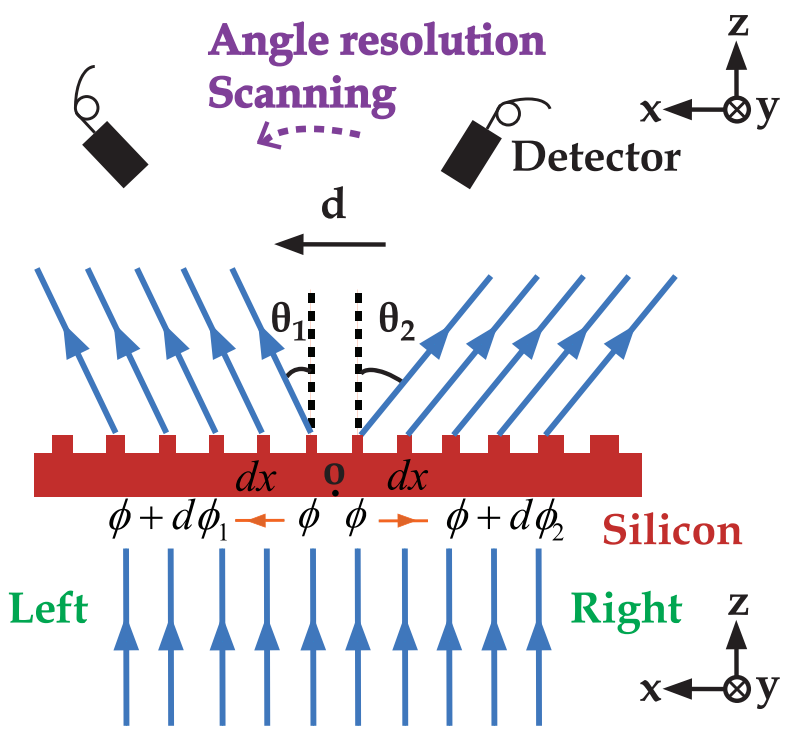

FIG. 1. Schematic of the all-dielectric metasurface-based beam splitter.

the refractive indices of the incident and refractive media, respectively; $d \phi_{1}$ and $d \phi_{2}$ are the phase changes along the left and right sides with respect to the point $O$. Suppose an $N$ step ( $N_{\text {left }}$ or $\left.N_{\text {right }}\right)$ phase gradient is adopted on the left or right side, Eqs. (2) and (3) thus can be simplified as

$$
\begin{aligned}
& n_{t} \sin \theta_{1}=n_{i} \sin \theta_{i}-\frac{c}{N_{\text {leff } f p}}, \\
& n_{t} \sin \theta_{2}=n_{i} \sin \theta_{i}+\frac{c}{N_{\text {right } f p}},
\end{aligned}
$$

where $f$ is the working frequency, $c$ is the speed of light, and $p$ is the horizontal period of the unit cells. If $f$ and $p$ are fixed, we can see that the angle of emergence $\theta_{1}\left(\theta_{2}\right)$ is dependent on the phase step $N_{\text {left }}\left(N_{\text {right }}\right)$. If we shift the metasurface along the left or right side, a variable SR thus could be obtained.

Let us consider a metasurface made of silicon cylinder on an isotropic silicon substrate. Figure 2(a) shows the schematic of a single silicon cylinder with a period of $p=150 \mu \mathrm{m}$ along both the $x$ and the $y$ axes. The thickness $t$ is $1.8 \mathrm{~mm}$, the height $h$ is fixed to $200 \mu \mathrm{m}$, and the radius $r$ is varied to get the desired phase. If $N=6$, the phase gradient has a $60^{\circ}$ phase interval, and the calculated angle of emergence is $28.43^{\circ}$ at $0.7 \mathrm{THz}$; while if $N=8$, the eight-step phase gradient has a $45^{\circ}$ phase interval with the angle of emergence of $20.92^{\circ}$ at $0.7 \mathrm{THz}$.

The computer simulation of the spectral response of the chosen unit cells was performed using the commercial software CST Microwave Studio. Figures 2(b) and 2(c) present the simulated transmission amplitude and phase of the sixstep and eight-step phase gradients of the selected unit cells with various $r$. Figures 2(d)-2(f) show the simulated electric field distributions at $0.7 \mathrm{THz}$ for three different designs: (1) $N_{\text {left }}=N_{\text {right }}=6$; (2) $N_{\text {left }}=N_{\text {right }}=8$; and (3) $N_{\text {left }}=6, N_{\text {right }}$ $=8$. The beam splitting is obviously observed from the simulated results for all three samples. Since the polarization of the observed electric field coincides with the incident polarization, the splitter is polarization independent.

To experimentally demonstrate our proposed designs, we fabricated the samples by convention photolithography combined with the deep reactive ion etching. A fiber-based angular resolved terahertz time-domain spectroscopy (THzTDS) is employed to characterize the transmission properties of the sample, as illustrated in Fig. 1. The basic setups of measurement are as follows: (1) The terahertz beam is
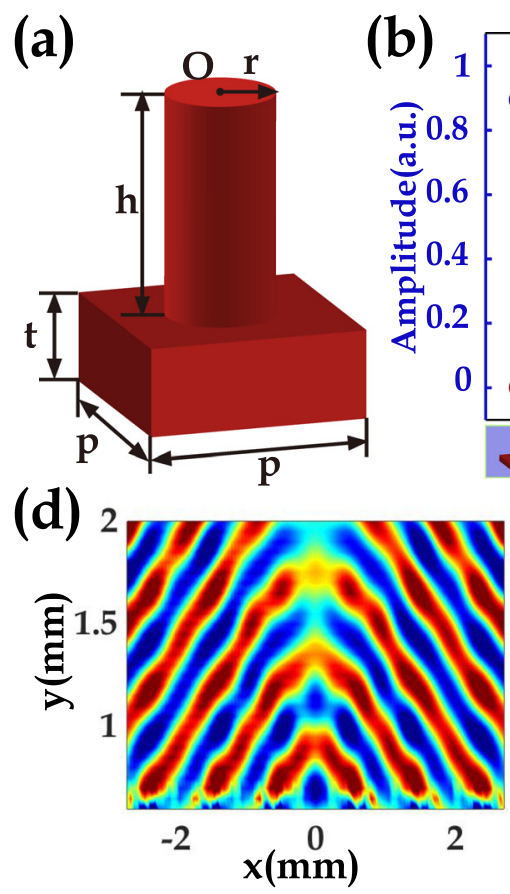

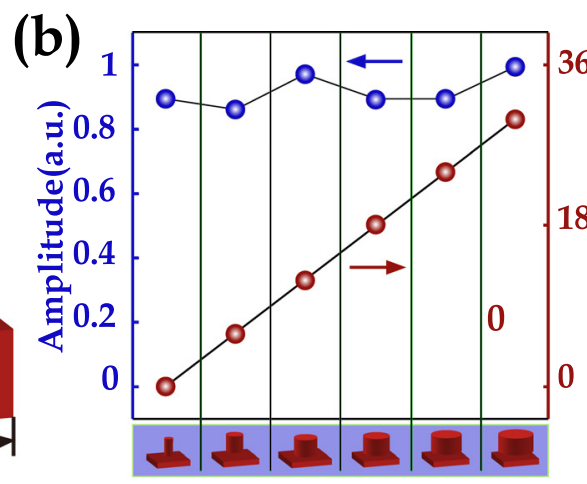

(e) 2

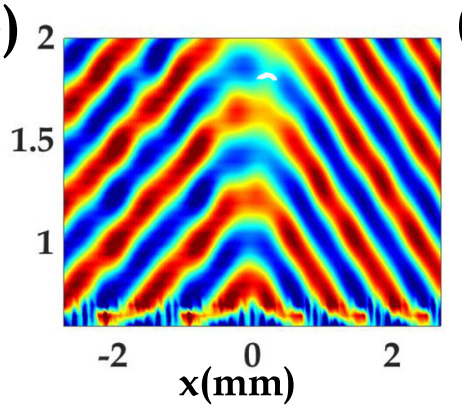

(c)

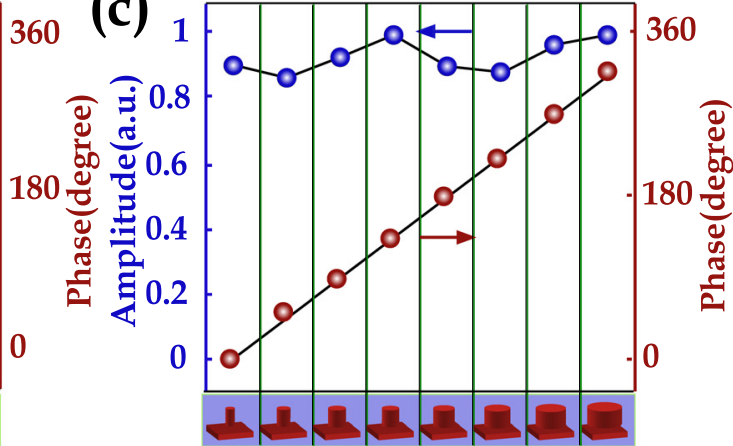

(f)

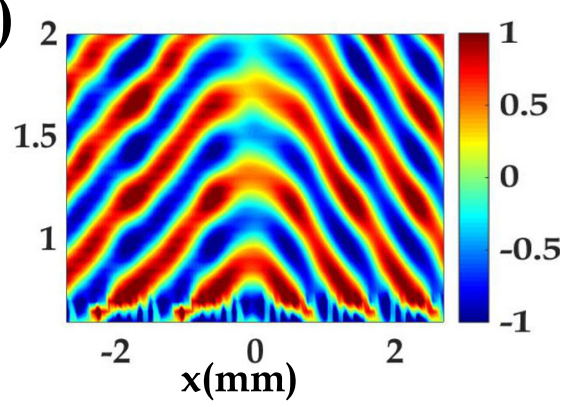

FIG. 2. (a) Schematic of the silicon cylinder: $p=150 \mu \mathrm{m}, t=1.8 \mathrm{~mm}$, and $h=200 \mu \mathrm{m}$. (b) and (c) Simulated transmission amplitude and phase of the unit cells for $N=6(r=33,42,47.75,54.25,63.5$, and $73.75 \mu \mathrm{m})$ and $N=8(r=33,40.25,44.75,49.5,54.25,61.5,68.5$, and 74.5 $\mu \mathrm{m})$ at $0.7 \mathrm{THz}$, respectively. (d) and (e) Simulated electric field distributions of the symmetric metasurface with $N_{\text {left }}=N_{\text {right }}=6$ and $N_{\text {left }}=N_{\text {right }}=8$, respectively. (f) Simulated electric field distributions of the asymmetric metasurface with $N_{\text {left }}=6$ and $N_{\text {right }}=8$. 

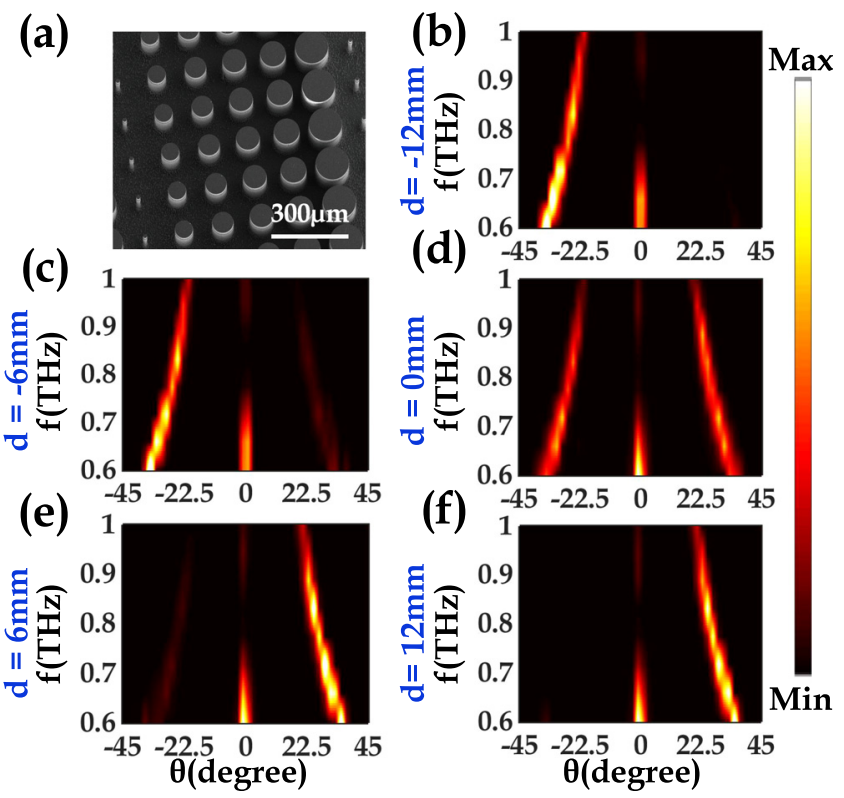

FIG. 3. (a) SEM (scanning electron microscopy) image of the sample. (b)-(f) Measured results of the beam splitter with $N_{\text {left }}=N_{\text {right }}=6$ under $x$ polarization with different $d$.

collimated by a lens; (2) The detector is placed $17.7 \mathrm{~cm}$ away from the sample; (3) A $6 \mathrm{~mm}$ aperture is applied before the detector to improve the resolution. The angular scanning is employed from $-45^{\circ}$ to $+45^{\circ}$, which is large enough to collect nearly all the terahertz signals. ${ }^{27}$ Besides, the sample is mechanically translated along the $+x$ or $-x$ direction.

Figure 3(a) shows the SEM image of the sample with $N_{\text {left }}=N_{\text {right }}=6$. The experimental results are shown with the increment of $6 \mathrm{~mm}$ in Figs. 3(b)-3(f), where the transmission spectra over the frequency range of $0.6-1 \mathrm{THz}$ are shown in a broad angular range from $-45^{\circ}$ to $+45^{\circ}$. The measured output angle at $0.7 \mathrm{THz}$ is $29.29^{\circ}$, agreeing well with the simulated result in Fig. 2. Although the metasurface is designed at $0.7 \mathrm{THz}$, it can work over a quite broad frequency band from 0.6 to $1 \mathrm{THz}$. What is interesting is that when we shift the sample from $d=-12$ to $12 \mathrm{~mm}$, the electric field intensity becomes weaker on the left side and becomes stronger on the right side. At $d=-12 \mathrm{~mm}$, the strong outgoing is observed at the left side as shown in Fig. 3(b); at $d=0 \mathrm{~mm}$, the symmetric outgoing is shown in Fig. 3(d); finally for $d=12 \mathrm{~mm}$, the left beam disappears, and the right one is the strongest as illustrated in Fig. 3(f). The center beam is due to the direct transmission through the metasurface. Meanwhile, the deflection angle shifts from $33.75^{\circ}$ to $19.47^{\circ}$ at from 0.6 to $1 \mathrm{THz}$ according to Eq. (4), which is caused by the dispersion nature of the metasurfaces. ${ }^{28}$ The corresponding angular dispersion can be calculated as follows: ${ }^{29} \frac{d \theta}{d \lambda}=\frac{\tan \theta}{\lambda}$, and such a dispersion can be eliminated or suppressed by using achromatic metasurface designs that reported recently. It is worth noting here that such a beam splitter is independent of the polarization state of the incident beam because the silicon cylinder is polarization independent, which can be seen clearly from the experimental measurements of our following another beam splitter.

Figure 4(a) shows the measured SR for various $d$ at $0.65,0.7$, and $0.95 \mathrm{THz}$. At the center point with $d=0 \mathrm{~mm}$, the measured SR is $1.07(48.31: 51.69)$ at $0.7 \mathrm{THz}$, where the (a)
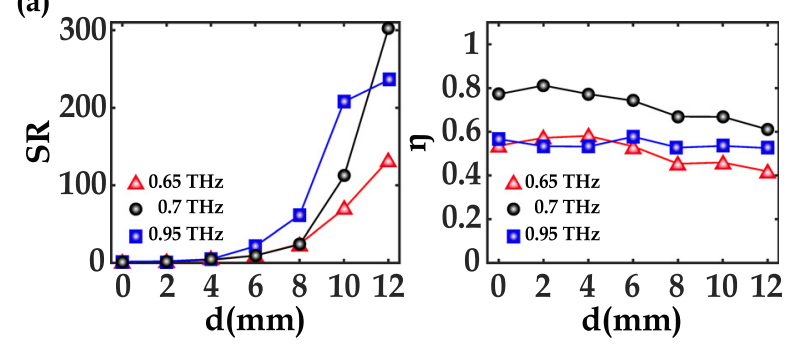

FIG. 4. (a) Measured SR of the beam splitter with $N_{\text {left }}=N_{\text {right }}=6$ under $x$ polarization for various $d$ at different frequencies: $0.65 \mathrm{THz}$ (triangles), $0.7 \mathrm{THz}$ (circles), and $0.95 \mathrm{THz}$ (squares). (b) Measured efficiency of the beam splitter with $N_{\text {left }}=N_{\text {right }}=6$ under $x$ polarization for various $d$ at different frequencies: $0.65 \mathrm{THz}$ (triangle), $0.7 \mathrm{THz}$ (circles), and $0.95 \mathrm{THz}$ (squares).

terahertz beam is divided into almost two equal beams. Shifting the beam splitter along the left side with $d$ changing from 0 to $6 \mathrm{~mm}$, as shown in Fig. 4(a), we see that the SR changes slowly. When $d=6 \mathrm{~mm}, \mathrm{SR}$ changes to 9.43 (9.59:90.41) at $0.7 \mathrm{THz}$. Further shifting the beam splitter along the left side, the SR changes dramatically. The SR is $301.6(0.33: 99.67)$ at $d=12 \mathrm{~mm}$ at $0.7 \mathrm{THz}$.

Additionally, we can define the conversion efficiency of our chosen beam splitters as

$$
\eta=\frac{I_{l e f t}+I_{\text {right }}}{I_{\text {ref }}}
$$

where $I_{\text {ref }}$ represents the electric field intensity of the reference (in our experiment, we choose a bare silicon substrate as the reference). Figure 4(b) shows that the measured efficiency for various $d$ at $0.65,0.7$, and $0.95 \mathrm{THz}$, respectively. At $0.7 \mathrm{THz}$ and $d=2 \mathrm{~mm}$, the maximal conversion efficiency is $81.22 \%$. If we defined the effective bandwidth as the conversion efficiency above $50 \%$, Fig. 4(b) shows that our proposed beam splitter has the bandwidth over $0.3 \mathrm{THz}$ ranging from 0.65 to $0.95 \mathrm{THz}$. Actually, we can further improve the conversion efficiency by several methods, such as using the geometric phase in reflective-type metasurfaces or high contrast meta-atoms. ${ }^{30,31}$

Figure 5 represented the measured results of another sample with the asymmetric design of $N_{\text {left }}=6$ and $N_{\text {right }}=8$, where both $x$ - and $y$-polarized incidences are measured. For various $d$, we found that the transmission response is almost identical for either $x$-polarized incidence or $y$-polarized incidence, indicating the non-polarizing characteristic of our proposed design. The measured angle of emergence is $30^{\circ}$ on the left side and is $20.82^{\circ}$ on the right side at $0.7 \mathrm{THz}$, which matches well with the simulated results in Fig. 2. As we shift the sample, the electric field distribution exhibits similar changing behaviors as those shown in Figs. 3(d)-3(f).

In practice, as there is little absorption of the silicon at terahertz, the thick silicon beam splitter uses the reflected and transmitted beams to split the incident beam, which thus has a high efficiency. In our proposed design, the reflected beam is not used, and the splitter efficiency of our device is lower than that of the thick silicon. In this case, it is better to choose the low refractive index substrate or coating antirefection layer at the bottom surface of the silicon. ${ }^{32}$ In addition, a thick silicon beam splitter normally would induce the 

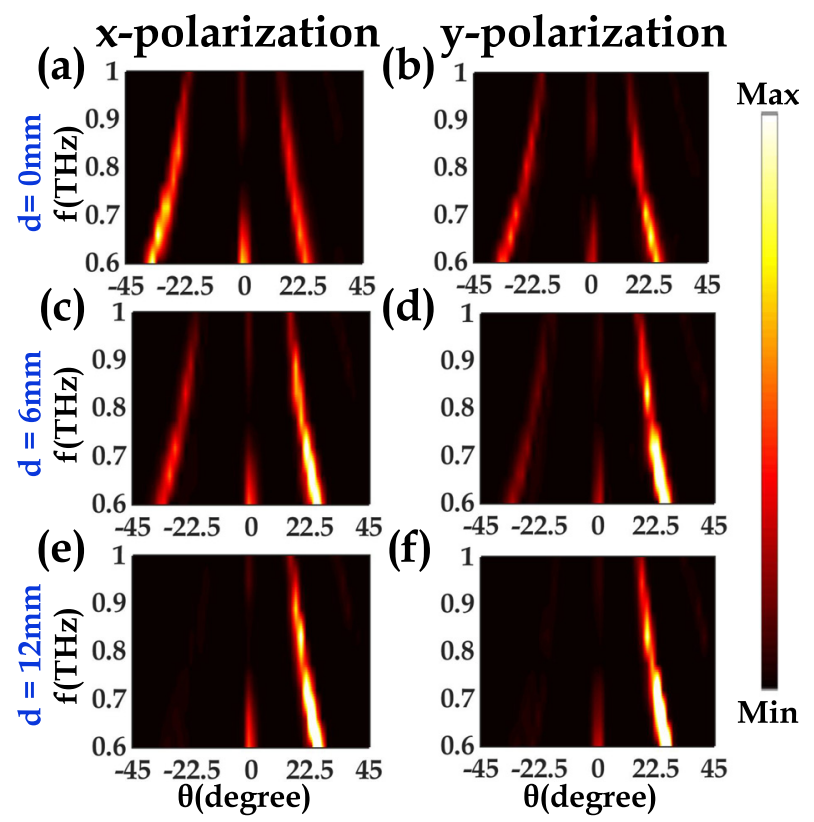

FIG. 5. (a)-(f) Measured results of the asymmetric polarization-independent beam splitter under $x$ and $y$ polarizations with different $d$.

time delay between the reflected and transmitted beams, which is not desired in some real applications. Moreover, the SR tunability of the silicon splitter is limited. By varying the incident angle, the split ratio of the silicon splitter can be tuned within some extent, but the corresponding refraction and reflection angles will be changed a lot accordingly, which is inconvenient for a lot of practical applications. For our proposed device, the emergent angles of two splitting beams can be flexibly manipulated by selecting different phase gradients.

In summary, an all-dielectric-metasurface-based terahertz beam splitter with a variable SR is proposed. The SR can be varied simply from 1.07 (48.31:51.69) to $301.6(0.33: 99.67)$ by mechanically moving the splitter toward one side. A broadband characteristic is also demonstrated over a range from 0.65 to $0.95 \mathrm{THz}$. Compared with the other methods, the proposed all-dielectric metasurface-based beam splitter is compact, non-polarizing, and easy-to-fabricate. The outgoing angles of the two split beams can be nearly arbitrarily designed, and its performance can be well kept under different polarization incidences. Although the work is demonstrated at terahertz frequencies, the proposed methodology is also applicable to the other frequency ranges.

This work was supported by the National Key Basic Research Program of China (Grant No. 2014CB339800), the National Science Foundation of China (Grant Nos.
61422509, 61420106006, and 61427814), and the U.S. National Science Foundation (Grant No. ECCS-1232081).

${ }^{1}$ X. Gao, J. H. Shi, X. Shen, H. F. Ma, W. X. Jiang, L. Li, and T. J. Cui, Appl. Phys. Lett. 102, 151912 (2013).

${ }^{2}$ H. J. Moo, R. H. Ho, P. S. Ryong, J. J. Wan, L. S. Gol, L. El-Hang, P. SeGeun, W. Deokha, K. Sunho, and O. Beom-Hoan, IEEE Photonics Tech. Lett. 15, 72 (2003).

${ }^{3}$ J. Feng and Z. Zhou, Opt. Lett. 32, 1662 (2007).

${ }^{4}$ Z. Wang, Y. Tang, L. Wosinski, and S. He, IEEE Photonics Technol. Lett. 22, 1568 (2010).

${ }^{5}$ H. Fukuda, K. Yamada, T. Tsuchizawa, T. Watanabe, H. Shinojima, and S.-I. Itabashi, Opt. Express 14, 12401 (2006).

${ }^{6} \mathrm{~K}$. Yang, X. Long, Y. Huang, and S. Wu, Opt. Commun. 284, 4650 (2011).

${ }^{7}$ M. Rahm, S. A. Cummer, D. Schurig, J. B. Pendry, and D. R. Smith, Phys. Rev. Lett. 100, 063903 (2008).

${ }^{8}$ M. Farmahini-Farahani and H. Mosallaei, Opt. Lett. 38, 462 (2013).

${ }^{9}$ H. Zhang, M. Kang, X. Zhang, W. Guo, C. Lv, Y. Li, W. Zhang, and J. Han, Adv. Mater. 29, 1604252 (2017).

${ }^{10}$ A. E. Costley, K. H. Hursey, G. F. Neill, and J. M. Ward, J. Opt. Soc. Am. 67, 979 (1977).

${ }^{11}$ C. W. Berry, J. Moore, and M. Jarrahi, Opt. Express 19, 1236 (2011).

${ }^{12}$ C. W. Berry and M. Jarrahi, J. Infrared, Millimeter, Terahertz Waves 33, 127 (2012).

${ }^{13}$ A. Rizea, Opto-Electron. Rev. 20, 96 (2012).

${ }^{14}$ N. Yu, P. Genevet, M. A. Kats, F. Aieta, J.-P. Tetienne, F. Capasso, and Z. Gaburro, Science 334, 333 (2011).

${ }^{15}$ N. M. Estakhri and A. Alù, J. Opt. Soc. Am. B 33, A21 (2015).

${ }^{16} \mathrm{P}$. Genevet, F. Capasso, F. Aieta, M. Khorasaninejad, and R. Devlin, Optica 4, 139 (2017).

${ }^{17}$ B. Scherger, C. Jördens, and M. Koch, Opt. Express 19, 4528 (2011).

${ }^{18}$ X. Y. Jiang, J. S. Ye, J. W. He, X. K. Wang, D. Hu, S. F. Feng, Q. Kan, and Y. Zhang, Opt. Express 21, 30030 (2013).

${ }^{19}$ L. Cong, W. Cao, X. Zhang, Z. Tian, J. Gu, R. Singh, J. Han, and W. Zhang, Appl. Phys. Lett. 103, 171107 (2013).

${ }^{20}$ L. Cong, N. Xu, J. Gu, R. Singh, J. Han, and W. Zhang, Laser Photonics Rev. 8, 626 (2014).

${ }^{21}$ H.-T. Chen, W. J. Padilla, M. J. Cich, A. K. Azad, R. D. Averitt, and A. J. Taylor, Nat. Photonics 3, 148 (2009).

${ }^{22}$ B. Sensale-Rodriguez, R. Yan, M. M. Kelly, T. Fang, K. Tahy, W. S. Hwang, D. Jena, L. Liu, and H. G. Xing, Nat. Commun. 3, 780 (2012).

${ }^{23}$ M. Khorasaninejad and F. Capasso, Nano Lett. 15, 6709 (2015).

${ }^{24}$ M. Khorasaninejad, W. Zhu, and K. B. Crozier, Optica 2, 376 (2015).

${ }^{25}$ Z. Zhou, J. Li, R. Su, B. Yao, H. Fang, K. Li, L. Zhou, J. Liu, D. Stellinga, C. P. Reardon, T. F. Krauss, and X. Wang, ACS Photonics 4, 544 (2017).

${ }^{26}$ X. Zhang, Z. Tian, W. Yue, J. Gu, S. Zhang, J. Han, and W. Zhang, Adv. Mater. 25, 4567 (2013).

${ }^{27}$ M. Wei, Q. Yang, X. Zhang, Y. Li, J. Gu, J. Han, and W. Zhang, Opt. Express 25, 15638 (2017).

${ }^{28}$ S. W. Qu, H. Yi, B. J. Chen, K. B. Ng, and C. H. Chan, Proc. IEEE 105, 1166 (2017).

${ }^{29}$ E. Arbabi, A. Arbabi, S. M. Kamali, Y. Horie, and A. Faraon, Optica 4, 625-632 (2017).

${ }^{30}$ Y. Yang, W. Wang, P. Moitra, I. I. Kravchenko, D. P. Briggs, and J. Valentine, Nano Lett. 14(3), 1394 (2014).

${ }^{31}$ A. Arbabi, Y. Horie, A. J. Ball, M. Bagheri, and A. Faraon, Nat. Commun. 6, 7069 (2017).

${ }^{32}$ H. Yi, S. W. Qu, K. B. Ng, C. H. Chan, and X. Bai, IEEE Trans. Antennas Propag. 64, 442 (2016). 